\title{
TUBERCULOSIS GANGLIONAR \\ RETROPERITONEAL Y MESENTERICA: CASO CLINICO
}

Drs. Rafael Martínez $F^{(1)}$, Paula Reyes $\mathbf{O}^{(1)}$, Giancarlo Schiappacasse $F^{(2)}$, Francisco Cruz $\mathrm{O}^{(3)}$, Antonieta Solar $G^{(4)}$.

1. Internos VII año Escuela de Medicina. Pontificia Universidad Católica de Chile.

2. Residente III año Radiología Pontificia Universidad Católica de Chile.

3. Médico Radiólogo Departamento de Radiología Pontificia Universidad Católica de Chile.

4. Anatómo-Patólogo Departamento de Patología Pontificia Universidad Católica de Chile.

\begin{abstract}
The changes of retroperitoneal lymph nodes in CT in a patient with AIDS are described. Differences with the compromise with $M$ aviumintracellulare are discussed an also the differential diagnosis with other lymph node pathologies.

Key words: Abdominal lymph nodes, AIDS, Disseminated Mycobacterium avium-intracellulare, Intraabdominal Mycobacterium tuberculosis, Tuberculous lymph nodes.
\end{abstract}

Resumen: Se describen las características del compromiso ganglionar por Mycobacterium tuberculosis en un paciente con SIDA en quien se demuestra alteraciones de linfonódulos retroperitoneales y mesentéricos en tomografía computada. Se discute las diferencias con el compromiso secundario a infección por Mycobacterium avium intracellulare y además el diagnóstico diferencial con otras formas de compromiso ganglionar.

Palabras claves: Linfonódulos abdominales, Mycobacterium Avium-intracelular, M. Tuberculoso, $T B C$ ganglionar, SIDA.

\section{Introducción}

Los informes del Ministerio de Salud para el año 2002 muestran una tendencia estacionaria de la tuberculosis (TBC) en Chile durante los últimos 3 años, con una tasa de prevalencia de $19.3 \times 100.000$ en este año; siendo la forma pulmonar la más significativa epidemiologicamente ${ }^{(1)}$.

La TBC intestinal y peritoneal es una

Martínez R. y cols. Tuberculosis ganglionar retroperitoneal y mesentérica: Caso clínico. Rev Chil Radiol 2004; 10:126-128.

Correspondencia: Rafael Martínez F. Avenida Grecia 4404 Ñuñoa, Santiago. Fono: 3268750/09-8178413. Email: rafamartinez@vtr.net enfermedad regional, crónica, específica, generalmente secundaria a TBC pulmonar significativa que adopta una forma localizada en el tejido linfoide local. El compromiso ganglionar es la manifestación más frecuente de la tuberculosis abdominal, siendo los linfonódulos mesentéricos, omentales y peripancreáticos los más frecuentemente afectados ${ }^{(2)}$.

La observación de un caso en un paciente con este último compromiso es motivo de esta presentación, destacando las características en imágenes del compromiso ganglionar y los diagnósticos diferenciales.

\section{Caso clínico}

Paciente de 59 años, del sexo masculino, con antecedentes de Diabetes Mellitus II diagnosticada desde hace un año. Consulta en el servicio de urgencia de nuestro hospital con historia de compromiso del estado general progresivo en los últimos 3 meses, baja de peso, y sudoración nocturna. La semana previa al ingreso se agrega dolor en flanco y fosa ilíaca izquierda, fiebre hasta $38,5^{\circ} \mathrm{C}$ y vómitos escasos.

Al examen físico se observa enflaquecido, pálido, febril, taquicárdico, destacando algorra en la cavidad bucal. Los diagnósticos de ingreso fueron: Síndrome febril, dolor abdominal en estudio, síndrome consuntivo, inmunodepresión, candidiasis oral.

Se realiza tomografía computada (TC) de abdomen demostrándose múltiples adenopatías retroperitoneales y a nivel de la raíz del mesenterio, algunas confluentes, hipodensas. Destacando después del uso de contraste una impregnación periférica (Figuras 1,2).

Posteriormente se confirma inmunodepresión asociada a VIH. Se realiza punción de adenopatías bajo TC; obteniéndose baciloscopía (+) para tuberculosis (TBC). La biopsia demuestra abundantes bacilos intracelulares (Figura 3). 


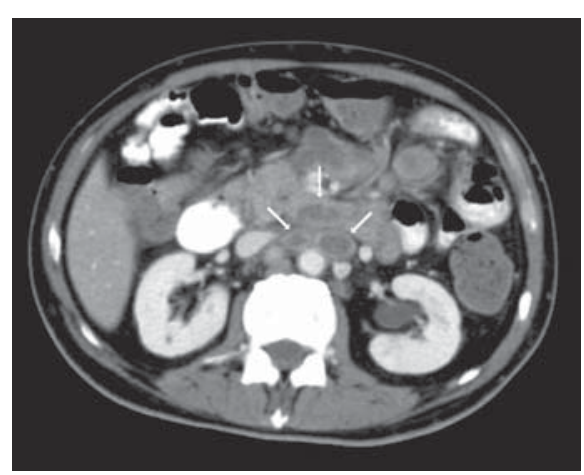

Figura 1. TC corte axial demostrando ganglios retroperitoneales que se impregnan periféricamente después de la inyección de medio de contraste (flechas).
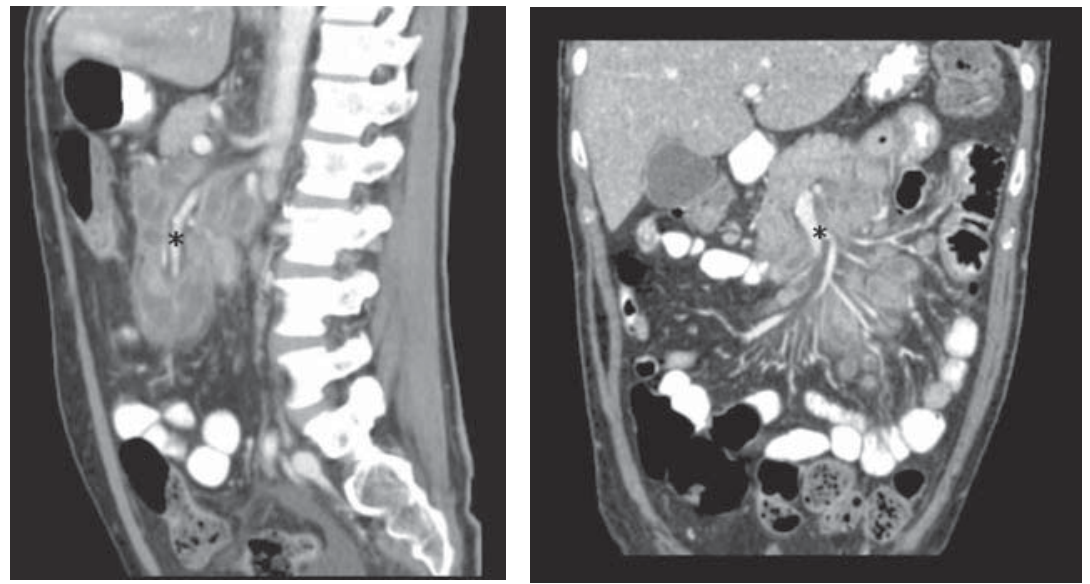

Figura 2 a,b. TC reconstrucción sagital (a) y coronal (b) demostrando adenopatías hipodensas, con impregnación periférica, rodeando la arteria mesentérica superior (asteriscos).

\section{Discusión}

EL compromiso ganglionar abdominal por TBC clínicamente se caracteriza por dolor, espontáneo y a la palpación, de localización difusa, a veces predominante en el cuadrante inferior derecho y asociado a náuseas y vómitos. Cuando existe compromiso de las asas intestinales puede haber alteración del tránsito, con disentería o constipación ${ }^{(2)}$.

Desde el punto de vista imagenológico se pueden plantear los diagnósticos diferenciales de: Linfoma, leucemia, adenopatías asociadas a infecciones granulomatosas tales como histoplasmosis, coccidioidomicosis, TBC y otras micobacteriosis (Micobacterium avium intracellulare). También se pueden considerar adenopatías en enfermedad de Whipple, hiperplasia ganglionar benigna en pacientes portadores de SIDA y metástasis ganglionares especialmente de tumores testiculares, del tracto genitourinario o sarcomas.

La presencia de adenopatías retroperitoneales imponen siempre un desafío para el radiólogo, quien en base a los antecedentes clínicos y las características imagenológicas debe proponer un razonable número de diagnósticos diferenciales que deben ser posteriormente confirmados mediante estudio histológico y eventualmente bacteriológico (cultivos, Gram, etc.).

El análisis de las adenopatías incluye: tamaño, número, ubicación, densidad e impregnación en TC.

El aumento de tamaño de los linfonódulos se establece en un límite que habitualmente se fija en $10 \mathrm{~mm}$ en el eje corto. Se pueden presentar en distintas ubicaciones, a veces en forma localizada o bien difusas y su número es variable, siendo en general múltiples. En ocasiones se presentan como grandes conglomerados, los que casi siempre sugieren una etiología maligna.

Las características de la impregnación con uso de contraste en TC a definir son; impregnación leve o significativa, periférica o central, homogéneo o heterogénea. De igual forma se debe precisar los coeficientes de atenuación que en la mayoría de los casos para TBC no supera las 30 unidades de Hounsfield (UH) sin contraste a diferencia del linfoma en donde es mayor ${ }^{(3,4)}$.

En el caso de nuestro paciente, se trataba de adenopatías múltiples, todas ellas mayores de 10 $\mathrm{mm}$, de ubicación principalmente retroperitoneal y mesentéricas, sin evidencia de conglomerados y que presentaban coeficientes de atenuación cercanos a $20 \mathrm{HU}$. Su comportamiento con el contraste intravenoso demostró impregnación preferentemente periférica. Estas características permitieron un diagnóstico diferencial más reducido en el cual se encontraban linfoma, adenopatías por TBC y Micobacterium avium intracellulare (MAI) y secundarias a compromiso metastático por neoplasias testiculares, del tracto urinario o de algunos sarcomas. En el contexto clínico del paciente, que considero condiciones sociales, edad, compromiso nutricional, evolución subaguda de los síntomas, ausencia de compromiso ganglionar fuera del abdomen y las características tomográficas, hizo proponer como primera posibilidad diagnóstica una etiología infecciosa, específicamente enfermedad por micobacterium, probablemente asociada a síndrome de inmunodepresión de base, sugiriéndose descartar infección por HIV.

Dentro de las infecciones por micobacterium las más frecuentes, en especial en pacientes inmunodeprimidos son TBC y MAI. Aun cuando el diagnóstico se establece mediante cultivos, han sido descritos hallazgos en TC que pueden permitir una diferenciación, situación no siempre fácil y por tanto sólo una aproximación diagnóstica.

El estudio realizado por Radin(5), en 71 pacientes con infección probada por M. Tuberculosis y MAI estableció características diferentes de los ganglios afectados. La presencia de necrosis central 


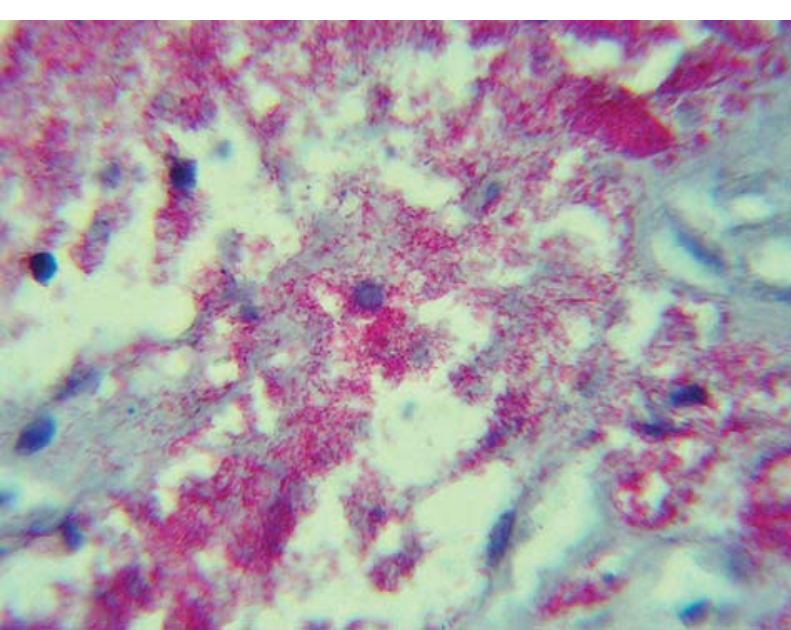

Figura 3. Muestra histológica obtenida por punción de uno de los ganglios demostrando la presencia de $M$. Tuberculoso.

o difusa se encontraba presente en un $93 \%$ de los pacientes con TBC versus un $14 \%$ de los afectados por MAI. Las diferencias en el compromiso de órganos sólidos, asas intestinales y peritoneo fueron de menor valor para el diagnostico diferencial sin embargo las lesiones focales en vísceras sugieren TBC y el crecimiento difuso del hígado y bazo mas el compromiso de la pared yeyunal sugieren MAI. Este estudio rebatió la información de publicaciones previas $^{(6-10)}$ que consideraban innecesaria la aspiración de adenopatías en pacientes con SIDA, y que consideraban que si ellas presentaban bajos coeficientes de atenuación en TC, tenían una alta probabilidad que fueran secundarias a infección por MAI. Este hecho era explicado por diferencias en el estado inmunitario del huésped y a la escasa respuesta con formación de granulomas caseificantes en pacientes con afectación por MAI en comparación a los afectados por M. tuberculosis.

Otro hecho de importancia que dejó de manifiesto este estudio es la relación de temporalidad entre infección por TBC versus MAl como enfermedad definitoria de etapa SIDA en paciente portadores de $\mathrm{VIH}$. Mientras el $85 \%$ de los primeros se presentaron con enfermedad diseminada como única patología, el $82 \%$ de los infectados por MAI tenían a lo menos otra enfermedad definitoria de SIDA, lo que traduce una menor virulencia de este último y su presentación por tanto en pacientes con mayor grado de compromiso inmunitario.

Debe considerarse que en pacientes con inmunodepresión, especialmente los afectados por
VIH, el diagnóstico diferencial también incluye linfoma, Sarcoma de Kaposi e hiperplasia ganglionar relacionado al VIH.

La mayor frecuencia de las infecciones por micobacterium, la baja atenuación de las adenopatías, su impregnación periférica, la ausencia de conglomerados y el mayor compromiso retroperitoneal y mesentérico sin afectación de otras localizaciones deben orientar principalmente al origen infeccioso de éstas.

\section{Conclusión}

Aunque son los estudios bacteriológicos e histológicos los esenciales para un diagnóstico definitivo, una adecuada orientación diagnóstica puede ser sugerida por el estudio de imágenes llevando a instaurar una terapia apropiada lo más pronto posible ayudando a un adecuado tratamiento.

\section{Bibliografía}

1. Situación epidemiológica de las enfermedades de notificación obligatoria. Reporte anual MINSAL 2002.

2. Harisinghani MG, Mc Loud TC, Shepard JA et al. Tuberculosis from head to toe. Radiographics 2000; 20: 449-470.

3. Pombo F, Rodríguez E, Caruncho MV et al. CT attenuation values and enhancing characteristics of thoracoabdominal lynphomatous adenopathies. J Comput Assist Tomogr 1994; 18: 59-62.

4. Pombo F, Rodriguez E, Mato J et al. Pattern of contrast enhancement of tuberculous lymph nodes demostrated by computed tomography. Clin Radiol 1992; 46: 13-17.

5. Radin DR. Intraabdominal Mycobacterium tuberculosis vs Mycobacterium avium-intracellulare infections in patients with AIDS: Distinction based on CT finding. AJR Am J Roentgenol 1991; 156: 487-491.

6. Hulnick DH, Megibow AJ, Naidich DP et al. Abdominal tuberculosis:CT evaluation. Radiology 1985; 157: 199-204.

7. Nyberg DA, Federle MP, Jeffrey RB et al. Abdominal CT findings in disseminated Mycobacterium aviumintracellulare in AIDS. AJR Am J Roentgenol 1985; 145: 297-299.

8. Jeffrey RB Jr, Nyberg DA, Bottles K, et al. Abdominal CT in adquired inmunodeficiency syndrome. AJR Am J Roentgenol 1986; 146: 7-13.

9. Federle MP. A radiologist looks at AIDS: imaging evaluation based on symptom complexes. Radiology 1988; 166: 553-562.

10. Lee J, Sagel S, Stanley R, Heiken J. Body TC correlation RM. Capítulo Retroperitoneo. 1999; 2: 1054-1068. 